\title{
KRAS mutation status impacts diagnosis and treatment decision in a patient with two colon tumours: a case report
}

\author{
Xiaodong Li, ${ }^{1}$ G Pezeshkpour, ${ }^{1,2}$ Ryan T Phan ${ }^{1,2}$
}

${ }^{1}$ Department of Pathology and Laboratory Medicine, USC/LAC Medical Center, Keck School of Medicine, University of Southern California, Los Angeles, California, USA ${ }^{2}$ Department of Pathology and Laboratory Medicine, VA Greater Los Angeles Healthcare System, Los Angeles, California, USA

\section{Correspondence to} Professor Ryan T Phan, Department of Pathology and Laboratory Medicine, VA Greater Los Angeles Healthcare System, 11301 Wilshire Blvd Bldg 500, Room 1255, Los Angeles, CA 90073, USA; Ryan.Phan@va.gov

Received 28 July 2014 Revised 18 September 2014 Accepted 24 September 2014 Published Online First 13 October 2014

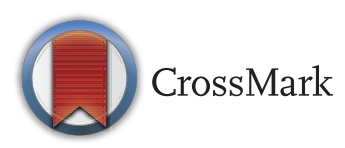

To cite: Li X Pezeshkpour G, Phan RT. J Clin Pathol 2015;68:83-85.

\section{ABSTRACT}

KRAS mutation status predicts response to anti-EGFR therapy in colorectal cancer patients. Here we report an interesting case of discordant KRAS mutation status in a patient with two separate tumour foci. Tumour A in sigmoid colon invaded through muscularis propria into the subserosal fat with metastatic disease in regional lymph nodes (pT3N2b). Tumour B in ascending colon had a relatively lower stage and no metastasis (pT2NO). Both tumours showed similar morphology, immunohistochemical staining and microsatellite instability pattern. KRAS mutation, however, was detected only in tumour $A$. These findings indicate distinct clonal nature of these two tumours. The discordance of KRAS mutation status also suggests that a combination of anti-epidermal growth factor receptor and chemotherapy is likely the best treatment option for this patient. This case exemplifies a notion that comprehensive pathological work-up comprising molecular testing is critical to guide the diagnosis and treatment decisions for colorectal cancer patients with multiple tumours.

\section{INTRODUCTION}

Colorectal cancer is the fourth leading cause of death worldwide causing approximately 608000 deaths in 2008 and $8 \%$ of all cancer related deaths. ${ }^{1}$ Besides surgery and traditional chemotherapy, anti-epidermal growth factor receptor (EGFR) antibodies such as cetuximab or panitumumab have routinely become treatment regimens either as a single agent or in combination with conventional chemotherapy for these patients. ${ }^{2}{ }^{3}$ However, patients with a colorectal tumour bearing a mutated KRAS gene fail to respond to anti-EGFR therapy. ${ }^{4-6}$ KRAS mutations are present in approximately $30 \%-50 \%$ of colorectal tumours. ${ }^{7}$ Testing for KRAS mutations is, therefore, recommended for colorectal cancer patients prior to the initiation of anti-EGFR therapy. ${ }^{4-6} 8$

Multiple tumours in colorectal cancer patients, either synchronous or metachronous, may be present as independent primaries or metastatic disease. In addition to histology and immunohistochemistry (IHC) staining, molecular testing is very useful in the pathology work-up of these cases. ${ }^{9} 10$ This study presents an interesting case in which different KRAS mutation status is identified in a colorectal cancer patient with two separate tumours of similar morphology, IHC pattern and even identical microsatellite instability (MSI) status.

\section{CASE PRESENTATION}

A patient with no family history of colon cancer presented for the follow-up of possible ulcerative colitis. Colonoscopy revealed a deep ulcer in the sigmoid colon and a single flat polyp in the ascending colon. Biopsies of each lesion were taken and adenocarcinoma was diagnosed in the specimen from the sigmoid colon, while malignancy could not be ruled out in the specimen from the ascending colon. A colectomy was performed and two tumours were identified during the procedure. The gross examination showed the first tumour (tumour A): a $4.7 \times 3 \mathrm{~cm}$, centrally ulcerated lesion with raised borders, located in the sigmoid colon; the second tumour (tumour B): a $1.5 \times 1.4 \mathrm{~cm}$, ulcerated lesion, located in the ascending colon. The distance between these two tumours was $96 \mathrm{~cm}$.

Histologically, both tumours exhibited well to moderately differentiated with glandular to solid architecture, and pleomorphic nuclei with prominent nucleoli (figure 1). However, tumour A was classified to be of more advanced stage with deep infiltration through the muscularis propria and involving the subserosal fat tissue. Lymph-vascular invasion was noted and metastatic adenocarcinoma was identified in 8 out of 36 regional lymph nodes (pT3N2b). In contrast, tumour B extended to but not through the muscularis propria without lymphvascular invasion. No metastasis was found in 27 regional lymph nodes (pT2N0). All the margins including proximal, distal, circumferential (radial) and vascular margin were negative for both tumours.

IHC was performed to further compare the morphology and investigate the nature of these two tumours. Tumour cells from both ascending and sigmoid colon showed a similar IHC pattern: weakly and focally positive for cytokeratin (CK)20, CDX-2, positive for MUC1, MUC3 and negative for CK7 and MUC2 in tumour A, with a weaker expression of CK 20 and CDX-2 in tumour B (table 1).

Molecular diagnostic tests were performed to provide insights to the pathological nature of the tumours. DNA was extracted and purified from paraffin embedded tissues obtained from these two tumours. The MSI analysis revealed identical MSI profiles in which both tumours A and B had four out of five tested microsatellite markers (D2S123, D17S250, D5S346 and BAT 26) stable and one microsatellite marker (BAT 25) unstable (table 2). These results indicate that both tumours A and B had low MSI (MSI-L). Additionally, BRAF and EGFR mutations were not detected in both 

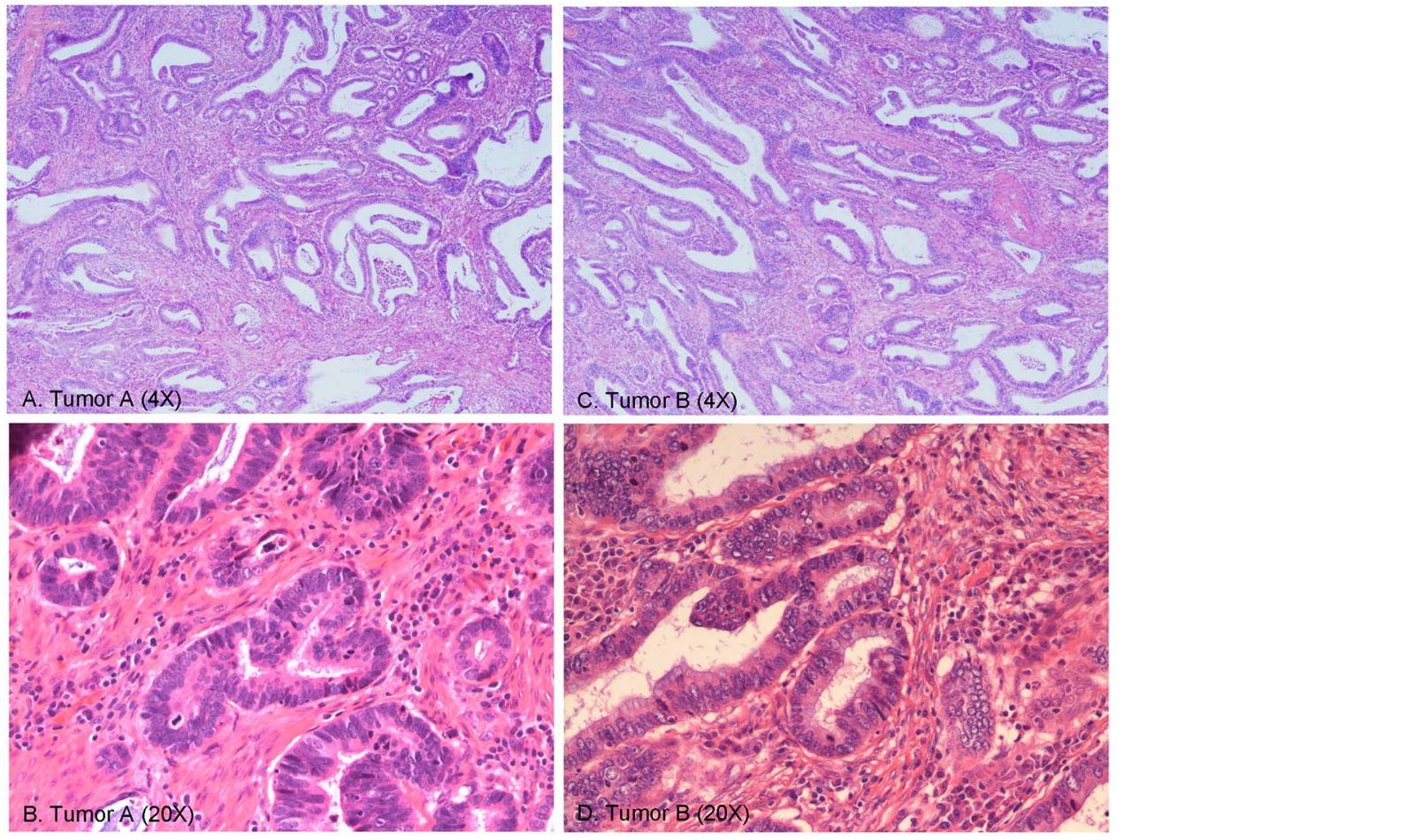

Figure 1 H\&E-stained sections of tumours: (A) tumour A (4x); (B) tumour A (20x); (C) tumour B (4x); and (D) tumour B (20x).

tumours A and B (data not shown). Molecular analysis, however, showed that a KRAS exon 2 point mutation $(\mathrm{GGT} \rightarrow \mathrm{GAT})$ was detected in tumour $\mathrm{A}$ and its regional lymph nodes whereas none of tested KRAS mutations were detected in tumour B (table 3). This mutation (GGT $\rightarrow$ GAT) occurs at codon 13 of the KRAS gene, resulting in a single amino acid substitution, glycine to aspartic acid.

To further investigate a possibility that tumour B may contain a few clones with KRAS mutation that are not detected due to clonal dilution, tumour B paraffin section was partitioned into four different parts for microdissection. Each part had approximately 65\%-85\% tumour content. KRAS testing was performed by real-time PCR using Taqman probes specifically recognised $K R A S$ wild-type and mutant alleles that can reliably detect KRAS mutation in as few as 5 mutated cells per 100 normal cells. The analysis revealed that no KRAS mutations are detected in all microdissected parts of tumour B (data not shown), confirming that the tumour $\mathrm{B}$ has no KRAS mutations.

\section{DISCUSSION}

A patient with multiple synchronous or metachronous tumours may have independent primaries or metastatic disease. These

Table 1 Immunohistochemical staining in tumours A and B

\begin{tabular}{lll}
\hline & Tumour A & Tumour B \\
\hline CK7 & - & - \\
CK20 & + & $+{ }^{w}$ \\
CDX-2 & + & $+{ }^{w}$ \\
MUC1 & + & + \\
MUC2 & - & - \\
MUC3 & + & + \\
\hline$+{ }^{w}$, weakly positive. & &
\end{tabular}

individual tumours with identical histological and IHC pattern are found to show different molecular aberrations. ${ }^{9}$ In the colon and rectum, incidence of multiple primary cancers is about $2 \%-5 \%{ }^{10}$ and the discordance of MSI status has been identified in individual tumours. ${ }^{11}$ Accordingly, these tumours may possess different genetic profiles including a different KRAS mutation status.

The risk for ulcerative colitis associated colorectal cancer is increased at least 2-fold compared with the normal population. Colorectal cancer is observed in 5.5\%-13.5\% of all patients with ulcerative colitis. ${ }^{12}$ These cancers typically can occur in the colon, are often multifocal ${ }^{12}$ and have the same incidence of KRAS mutation in comparison with sporadic colorectal cancer. ${ }^{13}$ In this study, both tumours A and B from this patient had a similar MSI profile (MSI-L). Together with the similarity of histological appearance and immunohistochemical pattern, these findings initially suggest that tumours A and B might have evolved from an identical tumour clone. Moreover, the tumour grade, stage and location also suggest that tumour B in ascending colon with relatively lower grade and stage is likely a metastasis from the more advanced tumour $\mathrm{A}$ in sigmoid, possibly through the lymphovascular spread.

A seminal study by Al-Mulla et $a l^{14}$ has previously reported $K R A S$ mutational heterogeneity in primary and secondary

Table 2 Microsatellite instability analysis in tumours A and B

\begin{tabular}{lll}
\hline Microsatellites & Tumour A & Tumour B \\
\hline D2S123 & Stable & Stable \\
D17S250 & Stable & Stable \\
D5S346 & Stable & Stable \\
BAT25 & Unstable & Unstable \\
BAT26 & Stable & Stable \\
\hline
\end{tabular}

Li X, et al. J Clin Pathol 2015;68:83-85. doi:10.1136/jclinpath-2014-202591 
Table 3 KRAS mutation analysis in tumours $\mathrm{A}$ and $\mathrm{B}$

\begin{tabular}{lll}
\hline Condon change & Tumour A & Tumour B \\
\hline Gly12Val & - & - \\
Gly12Asp & - & - \\
Gly12Ser & - & - \\
Gly12Cys & - & - \\
Gly12Arg & - & - \\
Gly12Ala & - & - \\
Gly13Asp & + & - \\
Gly13Val & - & - \\
Gly13Ser & - & - \\
Gly13Arg & - & - \\
Gly13Ala & - & - \\
Gly13Cys & - & - \\
\hline
\end{tabular}

colorectal carcinomas. Although the same KRAS mutations are found in the majority of patients with primary carcinomas and their metastases, many patients have a KRAS mutation in their primary carcinoma but none in liver metastases or patients have mutations in liver or lymph node metastases but none in the primary carcinoma, ${ }^{14}$ indicating that KRAS mutations are not always essential for the metastatic potential or might occur at the later stages of tumour progression. Importantly, Al-Mulla et al also reported a patient who has multiple adenomas with different KRAS mutations but only one of these mutations is found in corresponding liver metastasis, suggesting that these adenomas are independent neoplasms. ${ }^{14}$ In this context, the present case report is consistent with and in agreement with these findings. In fact, molecular analysis reveals that tumour A and metastatic regional lymph nodes contained a mutated KRAS gene whereas tumour B had a wild-type KRAS gene. This result, together with the findings of clear margins and no lymphvascular invasion in tumour $\mathrm{B}$, diminishes the possibility that tumour B is a metastasis from tumour A. Based on the patient's possible history of ulcerative colitis, these findings support the notion that these two tumours most likely originated from distinct clones and that both tumours possibly developed synchronously, with tumour A displaying a more advanced stage.

It is possible that the tumour B may evolve later to have the same KRAS mutation found in tumour A at a later stage of tumour progression. The present analysis, however, has no direct evidence to support this possibility.

This patient likely needs adjuvant therapy besides surgery due to the high tumour stage (pT3N2b). If only tumour A had been sampled for molecular testing, a KRAS positive result would exclude this patient from anti-EGFR therapy. As a result, tumour B would lose the benefit of anti-EGFR therapy. Likewise, if only tumour B had been tested and showed that it has KRAS wild-type, the patient would have been treated with an anti-EGFR regimen. Tumour A, carrying a KRAS mutation, would have failed to respond this treatment. ${ }^{4}{ }^{15}$ Unsuccessful outcome can be anticipated in either two scenarios. Instead, a combination of traditional chemotherapy and anti-EGFR therapy is likely the best treatment option with the best consequent prognosis for this patient. ${ }^{3}$

\section{CONCLUSIONS}

Traditional methods of histology and IHC are often insufficient to accurately anticipate the nature of the individual tumours in colorectal cancer patients with multiple primaries. This study demonstrates the importance of a comprehensive pathological work-up comprising molecular testing that impacts the diagnosis and guides treatment decisions.

\section{Take home message}

Molecular diagnostic tools for all the tumour sites provide insight to the pathological nature of the tumours and present a profound effect in the treatment decisions.

Acknowledgements We would like to thank Dr Faramarz Naeim and Dr Priyadashini Kumar for their critical review and comments on our manuscript.

Contributors XL, GP and RTP designed the study and performed the data analysis. $\mathrm{XL}$ and RTP organised the data for publication. XL and RTP wrote the manuscript.

\section{Competing interests None.}

Provenance and peer review Not commissioned; externally peer reviewed.

\section{REFERENCES}

1 Gimeno-Garcia AZ, Hernandez-Alvarez-Buylla N, Nicolas-Perez D, et al. Public Awareness of Colorectal Cancer Screening: Knowledge, Attitudes, and Interventions for Increasing Screening Uptake. ISRN Oncol. 2014. Vol 2014:425787.

2 Grothey A, Lenz HJ. Explaining the unexplainable: EGFR antibodies in colorectal cancer. J Clin Oncol 2012:30:1735-7.

3 Douillard JY, Oliner KS, Siena S, et al. Panitumumab-FOLFOX4 treatment and RAS mutations in colorectal cancer. N Engl J Med 2013;369:1023-34.

4 Karapetis CS, Khambata-Ford S, Jonker DJ, et al. K-ras mutations and benefit from cetuximab in advanced colorectal cancer. N Engl J Med 2008;359:1757-65.

5 Moorcraft SY, Smyth EC, Cunningham D. The role of personalized medicine in metastatic colorectal cancer: an evolving landscape. Therap Adv Gastroenterol 2013;6:381-95

6 Rizzo S, Bronte G, Fanale D, et al. Prognostic vs predictive molecular biomarkers in colorectal cancer: is KRAS and BRAF wild type status required for anti-EGFR therapy? Cancer Treat Rev. 2010;36(Suppl 3):S56-61.

7 Liu $X$, Jakubowski M, Hunt JL. KRAS gene mutation in colorectal cancer is correlated with increased proliferation and spontaneous apoptosis. Am J Clin Pathol 2011;135:245-52.

8 "Therascreen KRAS RGQ PCR Kit-P110030". Device Approvals and Clearances. U.S. Food and Drug Administration. 2012-07-06.

9 van der Sijp JR, van Meerbeeck JP, Maat AP, et al. Determination of the molecular relationship between multiple tumors within one patient is of clinical importance. J Clin Oncol 2002;20:1105-14.

10 Yoon JW, Lee SH, Ahn BK, et al. Clinical characteristics of multiple primary colorectal cancers. Cancer Res Treat 2008;40:71-4.

11 Bae JM, Cho NY, Kim TY, et al. Clinicopathologic and molecular characteristics of synchronous colorectal cancers: heterogeneity of clinical outcome depending on microsatellite instability status of individual tumors. Dis Colon Rectum. 2012;55:181-90.

12 Sanchez JA, Dejulius KL, Bronner M, et al. Relative role of methylator and tumor suppressor pathways in ulcerative colitis-associated colon cancer. Inflamm Bowel Dis 2011;17:1966-70.

13 Pohl C, Hombach A, Kruis W. Chronic inflammatory bowel disease and cancer. Hepatogastroenterology 2000;47:57-70.

14 Al-Mulla F, Going JJ, Sowden E, et al. Heterogeneity of mutant versus wild-type Ki-ras in primary and metastatic colorectal carcinomas, and association of codon-12 valine with early mortality. J of Pathology 1998;185:130-8.

15 Amado $\mathrm{RG}$, Wolf $\mathrm{M}$, Peeters $\mathrm{M}$, et al. Wild-type KRAS is required for panitumumab efficacy in patients with metastatic colorectal cancer. J Clin Oncol 2008;26:1626-34. 